\title{
Agendamento tático otimizado da colheita florestal em povoamentos de Pinus spp.
}

\section{Optimal harvest scheduling in Pinus spp. plantations for tactical planning}

\author{
Linamara Smaniotto Ferrari ${ }^{1}$, Julio Eduardo Arce $^{1}$ e Allan Libanio Pelissari ${ }^{1}$
}

\begin{abstract}
RESUMO
Conduziu-se este estudo com o propósito de avaliar o resultado de diferentes funções objetivo no agendamento tático da colheita florestal de Pinus spp., visando atender a demanda industrial, fatores econômicos e ambientais de uma empresa de celulose. A área de estudo foi de 3.714,68 hectares de florestas localizada no estado de Santa Catarina, Sul do Brasil. No processamento dos modelos matemáticos foram utilizadas variáveis de entrada baseadas em talhões e blocos, e testadas em quatro diferentes estratégias: (1) minimização de custos, (2) maximização do volume, (3) minimização da oscilação volumétrica ao longo do horizonte de planejamento e (4) minimização da dispersão das áreas de colheita. Os problemas foram resolvidos com base na programação linear inteira mista, seguindo a formulação do modelo Tipo I, para o sequenciamento anual das operações de colheita. O cenário que minimizou a oscilação do volume por blocos apresentou a melhor solução, uma vez que resulta numa produção equilibrada ao longo do horizonte de planejamento, limita custos de oportunidade relativas à compra de matéria-prima do mercado, minimiza perdas econômicas pertinentes às pausas no processo produtivo associadas ao deslocamento das frentes de trabalho, além de resultar em distribuição espacial dos blocos mais adepta em níveis operacional e ambiental. A abordagem de problemas operacionais de planejamento sem acréscimo de restrições espaciais nas funções matemáticas, mas com o apoio de um Sistema de Informações Geográficas, permitiu avaliar previamente a viabilidade dos resultados obtidos, oportunizando formulações menos complexas e que possam se ajustar às exigências ambientais pretendidas.
\end{abstract}

Palavras-chave: Planejamento florestal; Programação Linear Inteira Mista; Técnicas e Operações Florestais.

\begin{abstract}
This study evaluated the behavior of different objective functions of tactical scheduling in plantations Pinus spp., to meet the industrial demand; economic and environmental factors of a pulp company. The study area was 3,714.68 hectares of forest, located in the state of Santa Catarina, in southern Brazil. In the processing of the mathematical models, input variables based on stands and blocks were used and tested in four different strategies: (1) minimization of costs, (2) volume maximization, (3) minimization of volumetric oscillation over the planning horizon, and (4) minimization the distance between the work fronts in the harvesting operation. The problems were solved based on a model of mixed-integer linear programming following the formulation of the Type I model, for the annual sequencing of harvesting operations. The scenario that minimized the volume oscillation by blocks presented the best solution, since it results in a balanced production along the planning horizon, limits opportunity costs related to the purchase of raw material from the market, minimizes economic losses that are pertinent to pausing the productive process associated with the displacement of the work fronts, as well as reflecting a more balanced spatial distribution of the blocks, both at the environmental and operational levels. The approach to operational planning problems without adding spatial constraints to mathematical functions, but with the support of a Geographic Information System, has made it possible to evaluate the feasibility of the results obtained in advance, providing less complex formulations that can meet environmental requirements.
\end{abstract}

Keywords: Forest Planning; Mixed Integer Linear Programming; Techniques and Forest Operations.

\section{INTRODUÇÃO}

A base florestal brasileira é mundialmente reconhecida pela alta produtividade anual de suas áreas plantadas e pela menor rotação para corte final (IBÁ, 2017). Todavia, apesar da hegemonia mundial, nos últimos anos, a produção de madeira no país tornou-se mais cara. Os custos setoriais entre os anos de 2014 e 2015 cresceram 2,1 pontos percentuais a mais do que a inflação nacional,

1. Departamento de Ciências Florestais, Universidade Federal do Paraná - UFPR. Curitiba / PR, Brasil. Autor correspondente: linamara_ferrari@hotmail.com 
medida pelo Índice Nacional de Preços ao Consumidor (IBÁ, 2016). Por conseguinte, assegurar a competitividade da indústria florestal se torna um desafio, principalmente quando há dependência do mercado de exportação (RÖNNQVIST, 2003).

O planejamento, como um processo de tomada de decisão, exerce fundamental importância para a sustentabilidade dos empreendimentos florestais, tendo em vista a redução das incertezas (BANHARA, 2010). Apesar dos planos serem feitos em diferentes escalas, tais como estratégico, tático e operacional, esses devem ser coerentes à medida que se tornam mais específicos e com menor horizonte temporal de execução (BATEMAN; SNELL, 2012; MCDILL, 2014).

Uma das principais fases do planejamento da produção florestal se refere ao agendamento da colheita, pois envolve uma elevada representatividade no custo final do produto e possibilita a otimização das operações, bem como a minimização dos impactos ambientais (AUGUSTYNCZIK; ARCE; SILVA, 2015; MACHADO, 2014). Além de preocupações com a produção de madeira e a capitalização de recursos financeiros, nos últimos anos, as questões ambientais vêm sendo incorporadas nos planos de agendamento da colheita (GOMIDE, 2009). O cuidado com o ambiente tem se tornado um fator preponderante na sustentabilidade do mercado florestal, que vem reconhecendo a importância em mitigar o impacto ambiental da colheita em termos de erosão e estética da paisagem (MARQUES et al., 2014).

Na literatura, é possível encontrar uma série de estudos aplicados à problemas de agendamento florestal que avaliaram a distribuição espacial de áreas de corte relacionadas com fatores de abastecimento e produção, como: Augustynczik et al. (2015), Banhara et al. (2010), Chen et al. (2002); Gomide, Arce e Silva. (2010), Li, Bettinger e Boston (2010), McDill, Rebain; Braze (2002); Vielma et al. (2007) e Yoshimoto e Konoshima (2016). As práticas que envolvem distribuição espacial sugerem, tradicionalmente, o uso de restrições de adjacência espacial para limitar o tamanho da área de corte, cuja melhor maneira de lidar com esses objetivos no planejamento é incluí-los diretamente no problema de otimização (KURTTILA, 2001).

Todavia, essas estratégias de desenvolvimento exigem esforços adicionais de formulação, em que planos podem ser difíceis de serem implementados (BASKENT; JORDAN, 1996). Uma forma alternativa de considerar objetivos espaciais é encontrar, inicialmente, uma solução ideal para um modelo sem incluir restrições espaciais e, posteriormente, verificar a viabilidade da distribuição geográfica dos resultados obtidos (BETTINGER et al., 1996; KURTTILA, 2001). Assim, o presente estudo teve como propósito avaliar o comportamento de diferentes funções objetivo de agendamento tático da colheita florestal, em povoamentos de Pinus spp., que atendam a demanda industrial e os fatores econômicos e ambientais de uma empresa de celulose.

\section{MATERIAL E MÉTODOS}

\section{Área de estudo}

Este estudo foi desenvolvido com dados de povoamentos de Pinus spp. de uma empresa de celulose localizada no estado de Santa Catarina, região Sul do Brasil, que destina a matéria-prima ao abastecimento de sua planta. O ciclo de manejo, de plantio ao corte raso, é de 15 anos e sem a aplicação de desbaste. A área total considerada neste trabalho foi de 3.714,68 ha, sendo subdividida em 1.139 talhões, com idades de 10 a 15 anos (Figura 1).

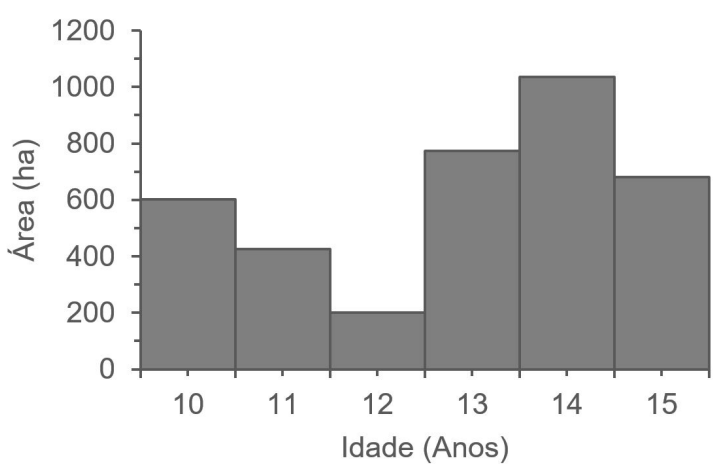

Figura 1. Distribuição inicial das classes de idade dos povoamentos de Pinus spp.

Figure 1. Initial distribution of the age classes of Pinus spp. stands. 


\section{Obtenção da matriz de distâncias}

Para o desenvolvimento do trabalho, foi necessário obter a matriz de distâncias entre os talhões e blocos, em que, para a formação dos blocos, os talhões foram agrupados em polígonos de mesma idade e localidade. Em situações que os talhões se apresentaram dispersos dos blocos e possuíam áreas menores que cinco hectares, eles foram unificados aos blocos mais próximos.

O centro de cada polígono foi considerado como o ponto de referência para talhões e blocos, visando determinar a matriz de distâncias via Sistema de Informações Geográficas (SIG). Para isso, utilizou-se a ferramenta centroide, com a opção Inside habilitada, do software ArcGIS versão 10.5 (ESRI, 2017).

Diante das várias possibilidades de caminhos que a frente de colheita pode adotar para se deslocar de uma unidade e outra, foi considerada a rota de menor distância entre as localidades, utilizando-se a extensão Network Analyst do software ArcGIS versão 10.5 (ESRI, 2017).

\section{Classificação de declividade}

Para efeitos de diferenciação dos custos operacionais obtidos na colheita florestal em diferentes declividades de terreno, a área de estudo foi classificada em duas categorias de relevo, de acordo com a prática adotada pela empresa e relacionada com os custos operacionais da colheita de madeira. Assim, talhões que apresentaram 50\% da área com declividade inferior a 15\% foram classificados como planos, ao passo que os demais foram considerados ondulados.

\section{Parâmetros}

Os sortimentos utilizados como padrão de processamento foram classificados como processo ( 8 a $28 \mathrm{~cm}$ de diâmetro com base na ponta fina e comprimento de 2,4 m) e toras (acima de $28 \mathrm{~cm}$ de diâmetro com comprimento de 2,6 m). O sortimento processo é utilizado unicamente para o abastecimento da indústria e todo excedente relacionado ao sortimento toras, quando existente, é destinado para venda no mercado, não havendo o compromisso de atender demanda específica desse produto. Ou seja, tem-se como objetivo abastecer à indústria de celulose e adicionalmente maximizar o resultado econômico do planejamento por meio da venda do sortimento excedente. Assim, as metas de produção anual foram estabelecidas para abastecimento da indústria, em um horizonte de planejamento de cinco anos, delimitado em 337.500 toneladas de madeira de processo para o primeiro ano e 450.000 toneladas para os demais quatro anos.

Foi considerado o fator 0,95 para conversão de metro cúbico para tonelada. Posteriormente, as estimativas de produção foram determinadas por meio do software SisPinus ${ }^{\circledR}$ (EMBRAPA, 2017), acionado internamente pelo OpTimber-LP, versão 2017. 4. 48 (OPTIMBER, 2017).

A taxa de juros foi fixada em $10 \%$ ao ano em todos os cenários simulados. Adicionalmente, como dados de entrada para o processamento dos cenários deste estudo, foram utilizados os preços e os custos praticados pela empresa no ano de 2016. Os custos operacionais da colheita de madeira foram, respectivamente, de $\mathrm{R} \$ 20,78$ e $\mathrm{R} \$ 25,70$ por tonelada de madeira cortada para áreas planas (abaixo de $15 \%$ de declividade) e onduladas (acima de 15\% de declividade). O levantamento dos custos de frete posto em fábrica foram aplicados apenas para o volume de processo, em que os valores variaram de acordo com a localidade de cada talhão e bloco, apresentando média de $\mathrm{R} \$ 7,84$ por tonelada. $\mathrm{O}$ valor utilizado para a compra de madeira de processo do mercado consistiu em $\mathrm{R} \$ 70,00$ por tonelada. Em contrapartida, o valor adotado para a venda de toras foi relativo à $\mathrm{R} \$ 132,50$ por tonelada.

Para a composição do cálculo dos custos presentes, considerados em cada simulação, utilizou-se o somatório do: custo de produção; custo de compra de madeira de processo do mercado; e, custo de frete.

\section{Modelo de otimização}

Com o uso de em um computador com processador Intel ${ }^{\circledR}$ Core $^{\mathrm{TM}}$ Duo CPU 2,93 GHz e 4Mb de memória RAM, o problema foi resolvido utilizando-se a Programação Linear Inteira Mista (PLIM), seguindo a formulação do modelo Tipo I (JOHNSON; SCHEURMANN, 1977), para o sequenciamento anual das operações de colheita das unidades selecionadas para o planejamento tático. O agendamento da colheita florestal requereu o uso de variáveis binárias $\{0,1\}$, devido a necessidade de colher totalmente uma unidade de manejo, conforme a exigência do regime adotado.

Diferentes cenários de otimização foram simulados para avaliar o desempenho do agendamento da colheita, sendo: Cenário A) Minimização dos custos por bloco [1]; Cenário B) Maximização do 
Ferrari et al. - Agendamento tático otimizado da colheita florestal em povoamentos de Pinus spp.

volume por bloco [2]; Cenário C) Minimização das oscilações de volume entre os anos consecutivos por bloco [3]; e, Cenário D) Minimização da dispersão da colheita florestal por bloco [4]. Adicionalmente, cada talhão foi considerado como um bloco nas funções objetivo, assim: Cenário E) Minimização dos custos por talhão [1]; Cenário F) Maximização do volume por talhão [2]; Cenário G) Minimização das oscilações de volume entre os anos consecutivos por talhão [3]; e, Cenário H) Minimização da dispersão da colheita florestal por talhão [4].

Todas as funções objetivo foram sujeitas à restrição [5], que garante a obtenção do volume mínimo demandado para o abastecimento da indústria ao longo do horizonte de planejamento, enquanto as equações [6], [7] e [8] foram aplicadas como restrições de singularidade, visando assegurar que as unidades de manejo sejam colhidas em sua totalidade. Dessa forma, os modelos de programação matemática empregados foram:

Função objetivo 1: Minimizar o custo presente geral, considerando o preço de compra e frete:

$$
\begin{aligned}
& \operatorname{MinZ}=\sum_{k=l l=l s=l}^{K} \sum^{L} \sum_{S=1}^{S}\left[(1+t)^{-k} \text { Compra }_{k l s} P C M_{k l s}\right] \\
& +\sum_{r=1 s=l}^{R} \sum_{s=1}^{S} \operatorname{Frete}_{r s} \sum_{r=I l=l}^{R} \sum_{l=l}^{L}\left[(1+t)^{-k} \text { YKLRS }_{k l r s}\right] \\
& +\sum_{i=1}^{I} \sum_{j=I}^{J}\left(C P_{i j} X_{i j}\right) \\
& C P_{i j}=\sum_{k=1}^{K}\left[\left(\left(R_{i j k}-C_{i j k}\right) /(1+t)^{-k}\right]\right.
\end{aligned}
$$

Em que: $i$ : Índice dos estratos a serem colhidos; $j$ : Índice do regime de manejo; $k$ : Período anual de planejamento; $l$ : Número de produtos florestais; $s$ : Número de destino; $r$ : Número de regiões; $B$ : Número total de blocos; J: Número total de regimes; $K$ : Número total de anos do horizonte de planejamento; $L$ : Número total de produtos florestais; $S$ : Número total de destinos; $R$ : Número de regiões; $t$ : Taxa anual de juros; Compra $_{k l s}$ : Volume de compra (ton) de madeira de terceiros do produto $l$, no ano $k$, para o destino $s ; P_{C M_{k l s}}$ : Custo [R $\$ /$ ton] de compra de madeira do produto $l$, no ano $k$, no destino $s$; YKLRS klrs $_{\text {: }}$ : Volume total (ton) de madeira do produto $l$, região $r$, destino $s$, no ano $k$; Frete $e_{r s}$ : Custo do frete $(\mathrm{R} \$)$ referente a região $r$ para o destino $s ; C P_{i j}$ : Custo de produção (R $\$ /$ ton) no estrato $i$ com regime $j ; R_{i j k}$ : Receita presente (R $\$ /$ ton) obtida com a venda de madeira de tora (diâmetro ponta fina de $28 \mathrm{~cm}$ e com comprimento de $2,6 \mathrm{~m}$ ) para terceiros referente ao estrato $i$, ano $k$, com regime $j ; C_{i j k}$ : Custo presente (R $\$$ /ton) obtido com a produção em madeira de todos os todos os estratos $i$, no ano $k$, com regime $j ; X_{i j}$ : Representa a área (ha) do povoamento no estrato $i$ com regime $j$.

Função objetivo 2: Maximizar a produção total de madeira para a indústria e venda para o mercado:

$$
\operatorname{Max} Z=\sum_{i=1}^{i \in r} \sum_{j=l}^{J} X_{i j} \sum_{k=l l=1}^{K} \sum_{i j k l} v_{i}
$$

Em que: $v_{i j k l}$ : Volume (ton/ha) potencial total existente nos estratos $i$, do produto $l$, no ano $k$, com regime $j$.

Função objetivo 3: Minimizar as oscilações de volume ao longo dos anos:

$$
\begin{aligned}
& \text { Min } Z=\text { MinMax-MaxMin } \quad \forall k \geq 2 \\
& \sum_{i=1}^{i \in r} \sum_{j=l}^{J} X_{i j} \sum_{k=l l=l}^{K} \sum_{i j k l}^{L} \leq \text { MinMax } \\
& \sum_{i=l}^{i \in r} \sum_{j=l}^{J} X_{i j} \sum_{k=l l=l}^{K} \sum_{i j k l}^{L} v_{i j l} \geq \text { MaxMin }
\end{aligned}
$$

Em que: MinMax: Menor das produções máximas (ton) possíveis no horizonte de planejamento; MaxMin: Maior das produções mínimas (ton) possíveis no horizonte de planejamento; MaxRaioColheita: Limite de distância $(\mathrm{Km})$ máxima de madeira.

Função objetivo 4: Minimizar a dispersão da colheita florestal

$$
\begin{aligned}
& \operatorname{Min} Z=\text { MaxRaioColheita } \\
& \text { DISTBLK }_{b l b 2} * \text { BINDISTBLK }_{b l b 2 k}<2 * \text { MaxRaioColheita } \quad \forall k \geq 1 \\
& \forall b 1, b 2 \quad b 2 \neq b 1 \\
& \text { BINBLK }_{b 1 k}+\text { BINBLK }_{b 2 k}-\text { BINDISTBLK }_{b 1 b 2 k} \leq 1 \quad \forall b 1, b 2 \quad b 2 \neq b 1
\end{aligned}
$$


Em que: DISTBLK $_{b l b 2}$ : Distância $(\mathrm{km})$ entre os blocos b1 e b2; BINDISTBLK $_{b l b 2 k}$ : Variável binária: 1, caso o trecho de estrada entre o par de blocos $\mathrm{b}=1 \mathrm{e} \mathrm{b}=2$ seja usado no ano $k ; 0$, caso contrário; $B I N B L K_{b l k}$ : Variável binária que assume valor 1 se o blocos $b 1$ seja escolhido para fazer parte da solução no ano $k$, ou valor 0 caso contrário; $B_{I N B L K_{b 2 k}}$ : Variável binária que assume valor 1 se o blocos $b 2$ seja escolhido para fazer parte da solução no ano $k$, ou valor 0 caso contrário.

Sujeito a:

$$
\begin{aligned}
& D E M A N D A_{k s}=\sum_{l=1}^{L} F T I K L S_{k l s} *\left(\sum_{r=1}^{R} Y K L R S_{k l r s}+\text { Compra }_{k l s}\right) \quad \forall k e s \\
& \sum_{s=1}^{S} Y K L R S_{k r r s}=\sum_{b} \sum_{j}^{i \in r}\left(X_{i j} v_{i j k l}\right) \\
& \sum_{k=1}^{K} B I N B L K_{b k} \leq 1 \\
& \sum_{i=l}^{i \in b j \in i} \sum_{j=l} X_{i j}+O C I O B L K_{b k}=\text { BINBLK }_{b k} * A R E A C O R T E B L K_{b k} \quad \forall b, \forall k
\end{aligned}
$$

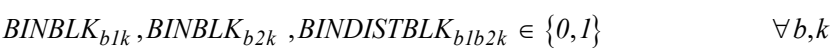

Em que: $D E M A N D A_{k s}$ : Demanda volumétrica (ton) em cada período do horizonte de planejamento $k$ e destino $s ; b$ : Índice dos blocos a ser colhido, considerando também um bloco para cada talhão nos Cenários E até $\mathrm{H} ; F_{T I K L S_{k l s}}$ : Fator de conversão $\mathrm{m}^{3}$ para toneladas; OCIOBLK $K_{b k}$ : Representa a área (ha) de corte ociosa no bloco b, no ano $k$; AREACORTEBLK ${ }_{b k}$ : Representa a área (ha) de corte no bloco $b$, no ano $k$.

\section{RESULTADOS E DISCUSSÃO}

As formulações dos modelos de planejamento que utilizaram blocos como parâmetros de agregação resultaram em 2.917 restrições e 36.253 variáveis de decisão, das quais 27.380 corresponderam a variáveis inteiras. Enquanto as estratégias que consideraram um bloco para cada talhão geraram 9.307 restrições e 6.506.128 de variáveis, sendo 6.486 .605 variáveis inteiras.

Os modelos resultaram em soluções viáveis para serem aplicadas em todas as estratégias avaliadas (Figura 2). Exceto para o Cenário H, que visou minimizar a dispersão da colheita em nível de talhão, apresentando insuficiência computacional para a resolução do problema.

Os cenários simulados utilizaram a área total disponível para suprir a demanda de volume ao longo do horizonte de planejamento. Todavia, é possível observar na Figura 2 que, em alguns anos, os cenários não mantiveram os níveis de produção mínima de volume para o abastecimento da indústria (demanda), tornando-se necessária a compra de matéria-prima do mercado.

Considerando as estratégias que minimizaram os custos por bloco (Cenário A) e por talhão (Cenário E), pode se constatar que houve maior concentração de corte no início do horizonte de planejamento, seguido de queda da produção nos dois últimos períodos. Essa tendência se evidencia pelo decréscimo da variação percentual do valor da floresta ao longo dos anos, até atingir o custo de oportunidade do capital (RODRIGUES, 1991; VIELMA et al., 2007), momento de máximo rendimento da floresta em relação à taxa de juros utilizada. Todavia, caso haja aumento do preço real de venda da madeira, a condição de corte raso pode resultar em maior idade de rotação, desde que o capital auferido com a venda possa ser aplicado à taxa utilizada (DIAZ-BALTEIRO et al., 2016).

Em contrapartida, os cenários que resultaram na máxima produtividade do povoamento (Cenários B e F), conforme apresentados na Figura 2, anteciparam a compra de madeira, de modo a manter as florestas em pé durante o maior tempo possível, visando obter o máximo volume do povoamento. Dessa maneira, pode-se observar que as aplicações das estratégias supracitadas apresentaram oscilação produtiva, bem como as unidades de manejo configuraram-se espacialmente dispersas ao longo do horizonte de planejamento (Figura 3).

A estratégia que minimizou a máxima dispersão da colheita florestal (Cenário D) apresentou a melhor distribuição dos blocos durante os cinco anos de planejamento (Figura 3). A incorporação de equações que buscam minimizar a distância da rede de estradas possibilita aumentar a eficiência das 
Ferrari et al. - Agendamento tático otimizado da colheita florestal em

povoamentos de Pinus spp.
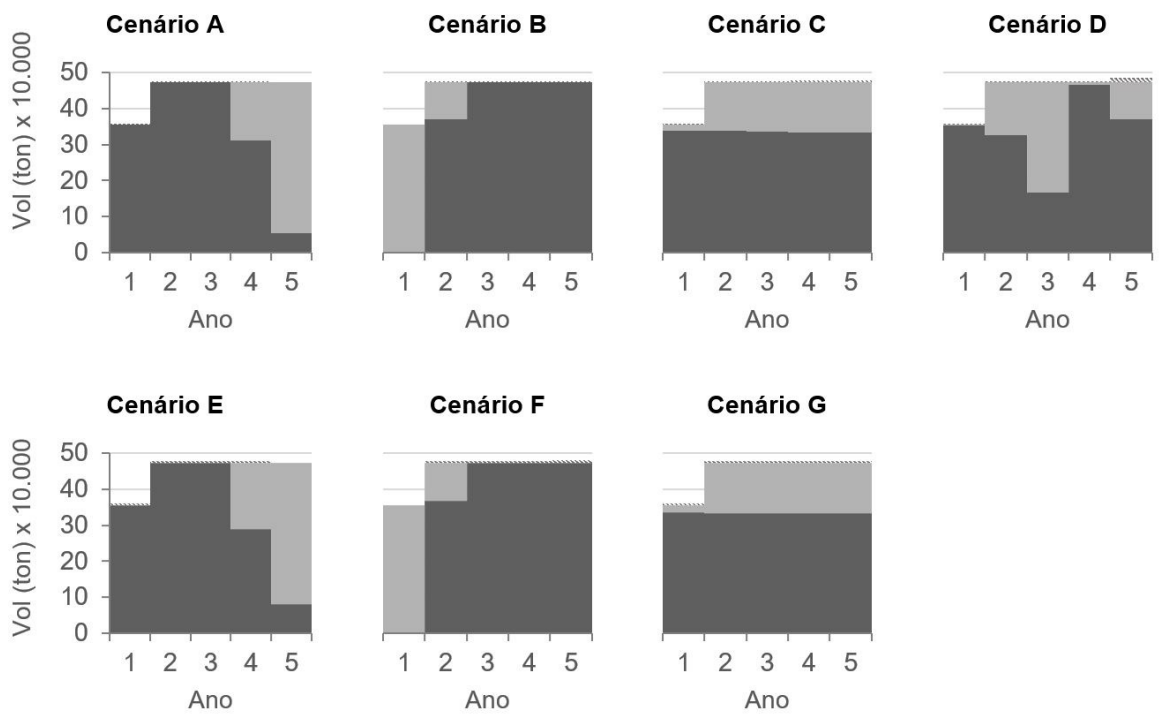

Cenário G

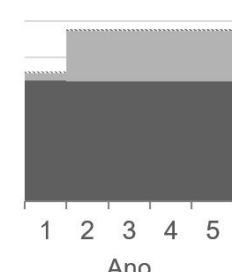

- Transferência indútria - Compra mercado : Venda mercado

Figura 2. Produção anual de madeira em cada ano do horizonte de planejamento.

Figure 2. Timber annual production for each year of the horizon planning.

operações e reduzir os impactos ambientais da colheita florestal, pois diminuem a movimentação das máquinas entre as frentes de colheita, minimizam o tempo improdutivo de realocação das máquinas e reduzem os custos relacionados à manutenção e contrução de estradas (AUGUSTYNCZIK, 2014; SILVA et al., 2016).

Todavia, embora o Cenário D possa ser favorável no âmbito econômico, contraria a combinação desejável de produção de recursos e de valores ambientais gerenciados em mosaicos na floresta. Os impactos gerados na paisagem após a colheita florestal são visíveis, podendo conter extensas áreas com exposição do solo, quebra da continuidade e retalhamento da paisagem, aumento de riscos causados por vendavais, dentre outros (GOMIDE et al., 2013). Além disso, a produção anual de madeira gerada nesse cenário (Figura 2) resultou em oscilações elevadas de volume a ser transferido da floresta para a fábrica, atingindo até $96 \%$ de diferença do volume a ser colhido entre o segundo e o terceiro ano.

Considerando os cenários que a função objetivo de equilíbrio volumétrico foi aplicada (Cenários Ce G), pode ser constatada que elas foram capazes de manter os níveis de produção durante o período considerado (Figura 2), embora tenham apresentado dispersão das unidades de corte (Figura 3). Além do mais, essas funções penalizaram a obtenção de resultados mais próximos ao da minimização dos custos, bem como em relação aos cenários que maximizaram a produção, uma vez que os pontos discrepantes característicos de cortes nos Cenários A, B, E e F foram equilibrados (Figura 2).

Dessa maneira, foi observado que a aplicação dos Cenários $\mathrm{C}$ e G geraram aumento dos custos totais em 3,1\% e 3,5\%, respectivamente (Tabela 1), em relação aos cenários que minimizaram custos. Entretanto, segundo Augustynczik et al. (2015), a manutenção dos níveis de produção durante o horizonte de planejamento é desejável do ponto de vista da empresa, bem como para os produtores florestais, pois garante a continuidade da produção e evita custos de oportunidade e perdas econômicas com interrupções no processo produtivo. Por conseguinte, pode-se observar que esses cenários atingiram a melhor combinação de produção de recursos e valores ecológicos, por meio do equilíbrio de volume e dispersão moderada da colheita em mosaico.

Avaliando o Cenário A em relação ao Cenário E (Tabela 1), observar-se que a estratégia que utilizou bloco como parâmetro de entrada resultou em custo levemente superior à estratégia que usou talhão, em decorrência da maior necessidade de compra de madeira de processo para o abastecimento da fábrica nos últimos anos. Em contrapartida, em relação aos Cenários B e F, que maximizaram o volume ao longo do horizonte de planejamento, houve maior custo no Cenário F que utilizou talhão como variável de entrada. 


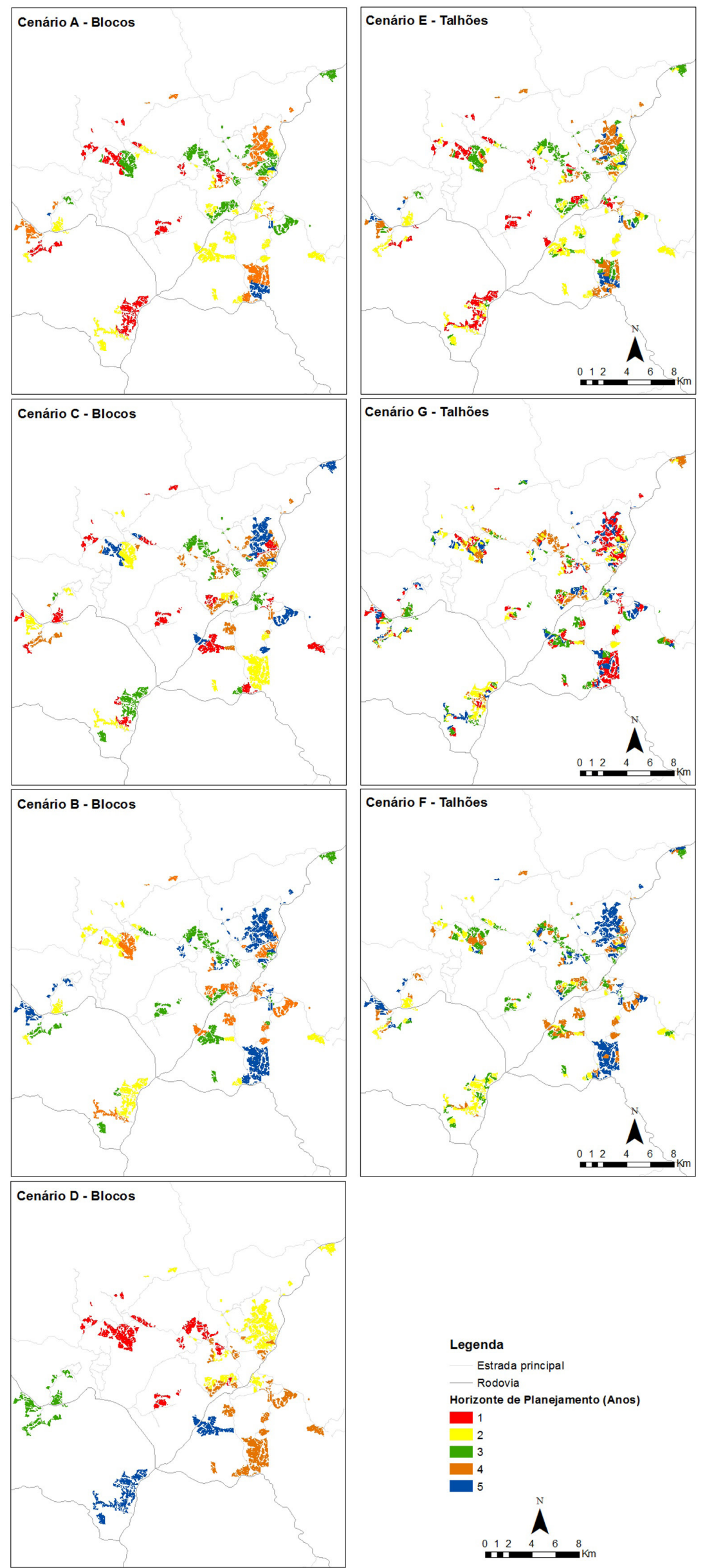

Figura 3. Agendamento espacial da colheita de Pinus spp. ao longo de cinco anos de planejamento. Figure 3. Spatial harvest scheduling of Pinus spp. over five years of planning. 
Ferrari et al. - Agendamento tático otimizado da colheita florestal em povoamentos de Pinus spp.

Tabela 1. Resultados do processo de otimização para cada cenário de agendamento espacial da colheita de Pinus spp.

Table 1. Optimization process results for each spatial harvest scheduling scenario of Pinus spp.

\begin{tabular}{|c|c|c|c|}
\hline Estratégias Blocos & $\begin{array}{l}\text { Custo presente } \\
(\mathrm{R} \$ \times 1.000 .000) \\
\end{array}$ & Estratégias Talhões & $\begin{array}{l}\text { Custo presente } \\
(\mathrm{R} \$ \times 1.000 .000)\end{array}$ \\
\hline Cenário $A$ & 64,20 & Cenário $\mathrm{E}$ & 64,10 \\
\hline Cenário $B$ & 65,78 & Cenário F & 65,90 \\
\hline Cenário C & 66,17 & Cenário G & 66,32 \\
\hline Cenário D & 66,44 & Cenário $\mathrm{H}$ & - \\
\hline
\end{tabular}

Essas diferenças no impacto do custo presente, em relação ao uso de blocos e talhões, estão associadas principalmente à dois motivos: necessidade de compra de madeira do mercado, que resulta em custo médio 55,6\% superior ao da produção acrescido com o valor do frete; e, relacionadas às características de integralidade utilizadas na formulação dos modelos, visto que um bloco quando agendado deve ser cortado de forma completa, obrigando que fragmentos com diferentes produtividades sejam colhidos em um mesmo ano.

Dessa maneira, o Cenário E apresentou menor custo que em relação ao Cenário A, em razão da seleção de unidades de áreas menores e com maior índice de homogeneidade. Isso garantiu o corte de áreas mais produtivas nos primeiros anos comparativamente ao Cenário $\mathrm{A}$, resultando, assim, em maior produção volumétrica, menor necessidade de compra de madeira do mercado e consequente redução dos custos.

Isso também explica os maiores custos do Cenário F em comparação ao Cenário B. Como suas funções objetivo consistiram em maximizar a produção total, o Cenário F foi capaz de manter em pé maior quantidade de unidades de área de maior produtividade durante um maior tempo possível, resultando na obtenção de maior estoque de madeira com diâmetro superior a $28 \mathrm{~cm}$ e menor quantidade de madeira de processo, ocasionando, assim, a necessidade de compra de madeira fina para o abastecimento da indústria e, sucessivamente, resultando em maior custo.

Além disso, os custos operacionais assumidos neste estudo consideraram as atuais práticas de colheita florestal utilizadas pela empresa. Sendo assim, a menor mobilização de máquinas, a menor necessidade de montagem de áreas de vivência e a minimização de manutenção e de construção de estradas podem resultar em custos operacionais menores. Com isso, os cenários que utilizam blocos como parâmetro de avaliação podem apresentar menores custos.

Em relação aos cenários que buscaram manter o fluxo volumétrico balanceado ao longo dos anos, obteve-se no Cenário $\mathrm{G}$ a necessidade de compra de 1,62\% de madeira de processo a mais que em relação ao Cenário $\mathrm{C}$. Esses cenários, diferentemente daqueles que minimizaram custos e maximizaram volume, não possuem uma tendência de seleção de áreas mais produtivas, e sim tendem a selecionar as áreas que resultem em uma menor diferença de volume a ser colhido ao longo do horizonte de planejamento.

Portanto, agregar unidades de manejo menores em áreas de maior escala de gerenciamento melhora a eficiência da colheita e transporte de madeira (YOSHIMOTO; KONOSHIMA, 2016), incluindo vantagens operacionais e logísticas por meio da concentração das áreas de corte da colheita (SMALTSCHINSKI; SEELING; BECKER, 2012). Todavia, o desenvolvimento de um conjunto abrangente de projetos que estejam funcionalmente relacionados e que combinem a produção de recursos juntamente com valores ambientais (BASKENT; JORDAN, 1996) ainda constitui-se um desafio fundamental para o agendamento espacial da colheita.

\section{CONCLUSÃO}

Dentre as estratégias abordadas neste estudo, recomenda-se principalmente o Cenário $\mathrm{C}$ de minimização das oscilações volumétricas entre anos consecutivos. Visto que esse cenário proporciona produção equilibrada ao longo do horizonte de planejamento, limita custos de oportunidade relativas à compra de matéria-prima do mercado, minimiza perdas econômicas pertinentes às pausas no processo produtivo associadas ao deslocamento das frentes de trabalho, além de resultar em distribuição espacial mais adepta em níveis operacional e ambiental.

Ressalta-se, também, que a abordagem adotada no Cenário $\mathrm{C}$, sem o acréscimo de restrições espaciais no problema e tendo como apoio um Sistema de Informações Geográficas, permite avaliar 
previamente a viabilidade dos resultados obtidos, o que oportuniza formulações matematicamente menos complexas e que possam se ajustar às exigências ambientais pretendidas.

\section{BIBLIOGRAFIA}

AUGUSTYNCZIK, A. L. D. Planejamento florestal otimizado considerando áreas mínimas e máximas operacionais de colheita. 2014. 170 p. Dissertação (Mestrado em Ciências Florestais) - Universidade Federal do Paraná, Curitiba, 2014.

AUGUSTYNCZIK, A. L. D.; ARCE, J. E.; SILVA, A. C. L. Planejamento espacial da colheita considerndo áreas máximas operacionais. Cerne, Lavras, v. 21, n. 4, p. 649-656, 2015.

BANHARA, J. R.; RODRIGUEZ, L. C. E.; SEIXAS, F; MOREIRA, J. M. M. A. P.; SILVA, L. M. S. da; NOBRE, S. R. N.; COGSWELL, A. Optimized harvest scheduling in eucalyptus plantations under operational, spatial and climatic constraints. Scientia Forestalis, v. 38, n. 85, p. 85-95, 2010.

BASKENT, E. Z.; KELES, S. Spatial forest planning: A review. Ecological Modelling, v. 188, n. 2-4, p. 145-173, 2005.

BASKENT, E. Z.; JORDAN, G. A. Designing forest management to control spatial structure of landscapes. Landscape and Urban Planning, v. 34, n. 1, p. 55-74, 1996.

BATEMAN, T. S; SNELL, S.A. Administração. 2. ed. Porto Alegre: AMGH, 2012. 408 p.

BETTINGER, P.; JOHNSON, K. N.; SESSIONS, J. Forest planning in an Oregon case study: Defining the problem and attempting to meet goals with a spatial-analysis technique. Environmental Management, v. 20, n. 4, p. $565-577,1996$.

DIAZ-BALTEIRO, L.; ROMERO, C.; RODRIGUEZ, L. C. E.; NOBRE, S. R.; BORGES, J. G. Economics and management of industrial forest plantations. In: BORGES, J. G.; DIAZ-BALTEIRO, L.; MCDILL, M. E.; RODRIGUEZ, L. C. The Management of industrial forest plantations: theoretical foundations and applications. Londres: Springer, 2014. p. 121-170.

EMBRAPA - EMPRESA BRASILEIRA DE PESQUISA AGROPECUÁRIA. Softwares para manejo florestal. 2017. Disponível em: < https://www.embrapa.br/florestas/transferencia-de-tecnologia/softwares-florestais >. Accesso em: 30 jan. 2017.

ESRI. ArcGis desktop: release 10.5. Redlands: Environmental Systems Research Institute, 2017.

GOMIDE, L. R. Planejamento florestal espacial. 2009. 235 p. Tese (Doutorado em Ciências Florestais) Universidade Federal do Paraná, Curitiba, 2009.

GOMIDE, L. R.; ARCE, J. E.; SILVA, A. C. L. da. Comparação entre meta-heurística simulated anneling e a programação linear inteira no agendamento da colheita com restrições de adjacência. Ciência Florestal, v. 23, n. 2, p. 449-460, 2013.

GOMIDE, L. R.; ARCE, J. E.; SILVA, A. L.. Efeito das restrições espaciais de adjacência no planejamento florestal otimizado. Revista Floresta, v. 40, n. 3, p. 573-584, 2010.

IBÁ - INDÚSTRIA BRASILEIRA DE ÁRVORES. Relatório Anual 2017: ano base 2016. São Paulo: IBÁ, 2017. 77 p. IBÁ - INDÚSTRIA BRASILEIRA DE ÁRVORES. Relatório Anual 2016: ano base 2015. São Paulo: IBÁ, 2016. 100 p.

JOHNSON, K. N.; SCHEURMANN, H. L. Techiniques for prescribing optimal timber harvest and investment under different objectives - discussion and synthesis. Forest Science, v. 18, n. 1, p. 1-31, 1977.

KURTTILA, M. The spatial structure of forests in the optimization calculations of forest planning - A landscape ecological perspective. Forest Ecology and Management, v. 142, n. 1-3, p. 129-142, 2001.

LI, R.; BETTINGER, P.; BOSTON, K. Informed development of meta heuristics for spatial forest planning problems. The open Operational Research Jornal, v. 4, p. 1-11, 2010.

MACHADO, C. C.; LOPES, E. S. Planejamento. In: MACHADO, C. C. Colheita Florestal. 3 ed. UFV, 2014. 543 p.

Sci. For., Piracicaba, v. 47, n. 121, p. 167-176, mar. 2019

DOI: dx.doi.org/10.18671/scifor.v47n121.17 
Ferrari et al. - Agendamento tático otimizado da colheita florestal em povoamentos de Pinus spp.

MARQUES, A. S.; AUDY, J. F.; D'AMOURS, S.; RÖNNQVIST, M. Tactical and operational harvest planning. In: BORGES, J. G.; DIAZ-BALTEIRO, L.; MCDILL, M. E.; RODRIGUEZ, L. C. The management of industrial forest plantations: theoretical foundations and applications. Londres: Springer, 2014. p. 239-267.

MCDILL, M. E. An overview of forest management planning and information management. In: BORGES, J. G.; DIAZ-BALTEIRO, L.; MCDILL, M. E.; RODRIGUEZ, L. C. The management of industrial forest plantations. Springer. 2014. p. 27-59.

MCDILL, M. E.; REBAIN, S. A.; BRAZE, J. Harvest scheduling with area-based adjacency constraints. Forest Science, v. 48, n. 4, p. 631-642, 2002.

OPTIMBER OTIMIZAÇÃO E INFORMÁTICA LTDA. Produtos. 2017. Disponível em:< http://www.optimber. com.br/index.php/softwares/optimber-lp >. Acesso em: 30 jan. 2017.

RODRIGUES, L. C. E. Gerenciamento da produção florestal. Documentos Florestais. Piracicaba: Universidade de São Paulo, Escola Superior de Agricultura Luiz de Queiroz, Departamento de Ciências Florestais, v.13, p.141, 1991.

RÖNNQVIST, M. Optimization in forestry. Mathematical programming, v. 97, n. 1-2, p. 267-284, 2003.

SILVA, P. H. B. M.; ARCE, J. E.; LOCH, G. V.; DAVID, H. C.; FIORENTIN, L. D. Forest harvest scheduling plan integrated to the road network. Cerne, v. 22, n. 1, p. 69-76, 2016.

SMALTSCHINSKI, T.; SEELING, U.; BECKER, G. Clustering forest harvest stands on spatial networks for optimised harvest scheduling. Annals of Forest Science, v. 69, n. 5, p. 651-657, 2012.

VIELMA, J. P.; MURRAY, A. T.; RYAN, D. M.; WEINTRAUB, A. Improving computational capabilities for addressing volume constraints in forest harvest scheduling problems. European Journal of Operational Research, v. 176, n. 2, p. 1246-1264, 2007.

YOSHIMOTO, A.; KONOSHIMA, M. Spatially constrained harvest scheduling for multiple harvests by exact formulation with common matrix algebra. Journal of Forest Research, v. 21, n. 1, p. 15-22, 2016.

Recebido em: 28/09/2017

Aceito em: 19/07/2018 